\title{
FATORES ASSOCIADOS À INTENSIDADE DAS ONDAS DE CALOR EM MULHERES EM CLIMATÉRIO
}

\author{
Danielle Santos-Sá, Aarão Mendes Pinto-Neto*, Délio Marques Conde, Adriana Orcesi Pedro, Simone Caetano Morale de Oliveira, \\ Lúcia Costa-Paiva \\ Trabalho realizado no Departamento de Tocoginecologia, Faculdade de Ciências Médicas, Universidade Estadual de Campinas (SP)
}

*Correspondência:

Rua Alexander Fleming, 101

Cidade Universitária

"Zeferino Vaz"

Cep: $13.083-970$

Campinas, SP

Fone/Fax: (19) 3788-9306

aarao@unicamp.br

\section{RESUMO}

OBjetivo. Identificar os fatores associados à intensidade das ondas de calor em mulheres climatéricas em Campinas, São Paulo.

Métodos. Análise secundária de banco de dados de estudo descritivo de coorte transversal, de base populacional. Foram selecionadas 334 mulheres com ondas de calor, por amostragem, entre 45-60 anos. Os dados foram coletados por meio de entrevistas domiciliares, com questionários estruturados e pré-testados, fornecidos pela Fundação Internacional de Saúde/ Sociedade Internacional de Menopausa, Sociedade Norte-Americana de Menopausa, e adaptados pelos autores, e a intensidade das ondas de calor mensurada por meio do índice circulatório. Avaliou-se a idade, uso de métodos anticoncepcionais, terapia hormonal, laqueadura tubária, índice de massa corpórea, estado menopausal, tempo de menopausa, antecedente de histerectomia, ooforectomia bilateral e tabagismo. Calculou-se a mediana e as frequêencias absolutas e relativas, de acordo com o tipo de variável. A medida de associação foi a razão de prevalência (RP). Realizou-se análise bivariada e de regressão múltipla para identificação dos fatores associados à intensidade das ondas de calor, com intervalo de confiança de 95\% (IC 95\%).

Resultados. Mulheres com tempo de menopausa superior a 6 I meses (RP: 0,59; IC $95 \%$ : 0,39-0,88) apresentaram chance significativamente menor de referir ondas de calor intensas enquanto 0 antecedente de ooforectomia bilateral (RP: I,95; IC 95\%: 1,08-3,50) associou-se significativamente à intensidade das ondas de calor.

Conclusäo. Os fatores associados às ondas de calor de maior intensidade, tempo de menopausa e ooforectomia bilateral sugerem o hipoestrogenismo como causa comum para as ondas de calor intensas. Mulheres com esses fatores devem receber atenção especializada, minimizando as repercussões negativas das ondas de calor.

UnITERMOS: Menopausa. Estudos transversais. Ondas de calor.

\section{INTRODUÇÃO}

As ondas de calor são queixas comuns referidas por mulheres durante a transição climatérica. Conceitualmente, são definidas como períodos transitórios de intenso calor na parte superior do corpo, braços e face, seguidos de enrubecimento da pele e sudorese profusa. São acompanhadas de palpitações e de sensação de ansiedade!. As ondas de calor freqüentemente determinam um impacto negativo na qualidade de vida, relacionando-se a alterações do sono com conseqüente fadiga, irritabilidade, desconforto físico e problemas no trabalho².

No Brasil, segundo estudo de base populacional, a prevalência de ondas de calor é de 70,3\% em mulheres no climatério ${ }^{3}$. Embora pareça ter como principal causa a deficiência estrogênica, as ondas de calor apresentam prevalência e intensidade que variam segundo características da população estudada. Dessa forma, o índice de massa corpórea (IMC), tabagismo, consumo de álcool, escolaridade, antecedente de ooforectomia ou de câncer são alguns fatores associados à intensidade das ondas de calor 4,5 . Os dados do Study of Women's Health Across the Nation (SWAN), realizado nos Estados Unidos, demonstraram que a prevalência de ondas de calor apresenta diferenças segundo a cor/etnia. As ondas de calor foram mais comuns em mulheres afro-americanas (45,6\%), seguidas por hispânicas (35,4\%), caucasianas $(31,2 \%)$, chinesas $(20,5 \%)$ e japonesas $(17,6 \%)$. Observou-se uma associação significativa entre índice de massa corpórea, tabagismo, atividade física, cor/etnia, estado menopausal e ondas de calor ${ }^{5}$.

Estudo de base populacional reportou prevalência de $75 \%$ de ondas de calor em mulheres australianas ${ }^{6}$. Nesse estudo, observou-se que as ondas de calor associaram-se ao estado de pós-menopausa, com alta prevalência dois anos antes da menopausa, atingindo um pico dois anos após a última menstruação. Em outro estudo de base populacional, o Massachusetts Women's Health Study, a prevalência de ondas de calor foi de 10\% na pré-menopausa (ciclos menstruais regulares) e de aproximadamente $40 \%$ na perimenopausa (ciclos menstruais irregulares). Verificou-se que mulheres na perimenopausa relataram maior freqüência de consultas médicas, o que pode sugerir uma maior intensidade dos sintomas nesse período, determinando um maior prejuízo à vida dessas mulheres ${ }^{5}$.

O presente estudo é parte de um estudo de base populacional que avaliou mulheres com idade entre 45 e 60 anos, 
na cidade de Campinas, São Paulo³. Observou-se inicialmente que as ondas de calor são uma das principais causas de procura por serviços médicos e são os sintomas vasomotores mais prevalentes, porém a intensidade desses sintomas (ondas de calor, sudorese, tontura, palpitação) não variou ao longo da pré-menopausa, perimenopausa e pós-menopausa ${ }^{3}$. Não foram identificados fatores que isoladamente associaram-se à ocorrência das ondas de calor ${ }^{7}$. Após os estudos iniciais, permaneceu a dúvida de quais fatores poderiam associar-se à intensidade das ondas de calor, uma vez que aquelas de maior intensidade podem comprometer a qualidade de vida.

Considerando-se as repercussões negativas das ondas de calor sobre a qualidade de vida, a influência de fatores socioculturais na sua prevalência e intensidade e a inexistência de estudos nacionais de base populacional abordando o tema, conduziu-se o presente estudo. O objetivo foi identificar os fatores associados à intensidade das ondas de calor por meio de um inquérito populacional domiciliar com a finalidade de contribuir para a discussão do tema e minimizar a influência desses fatores sobre as ondas de calor.

\section{Métodos}

Realizou-se análise secundária de um banco de dados de um estudo descritivo de coorte transversal e exploratório, tipo inquérito populacional. A metodologia deste estudo foi previamente publicada ${ }^{3}$. De forma breve, a coleta de dados foi iniciada em outubro de 1997 e encerrada em janeiro de 1998, totalizando 73 dias de trabalho de campo. O tamanho da população alvo considerada para o cálculo do tamanho amostral foi a população feminina de Campinas, na faixa etária entre 45 a 60 anos, no ano de 1997. O número mínimo calculado foi de 367 mulheres, mas foi acrescido de 20\%, resultando em um número de 456 entrevistas, para contemplar as possíveis usuárias de terapia hormonal. Este acréscimo se justifica porque, na análise estatística do estudo inicial, as mulheres em uso de anticoncepcionais hormonais ou de terapia hormonal foram excluídas da análise da prevalência de sintomas, visto que essas medicações poderiam mascará-los ${ }^{3}$.

Nesta análise secundária sobre a intensidade das ondas de calor, foram incluídas apenas as mulheres que referiram ondas de calor $(n=334)$, entre 45 e 60 anos de idade, residentes em Campinas, São Paulo, brasileiras natas. Um questionário estruturado e pré-testado foi utilizado para obter as informações, tendo sido elaborado a partir de dois questionários adaptados pelos autores. O primeiro questionário foi fornecido pela Sociedade Internacional de Menopausa e Fundação Internacional de Saúde, após ter sido aplicado em sete países do Sudeste Asiático. O segundo questionário foi fornecido pela Sociedade NorteAmericana de Menopausa e foi aplicado nos Estados Unidos ${ }^{8}$.

Um Termo de Consentimento Livre e Esclarecido, redigido conforme a Resolução 196/96 do Conselho Nacional de Saúde, foi lido para as participantes do estudo, não sendo solicitada a assinatura de um formulário de consentimento informado. Somente o consentimento oral foi necessário.
A variável dependente considerada foi a intensidade das ondas de calor. Para a avaliação da intensidade desse sintoma, foi adotado o índice circulatório, que expressa valores médios para a frequêencia das ondas de calor referida pelas mulheres. Este índice é obtido pela adição dos escores da frequiência de ondas de calor indicada pelas mulheres, variando de I (nunca) a 4 (I I vezes ou mais por dia) $)^{8}$. Dessa forma, as ondas de calor foram categorizadas segundo o índice circulatório em dois grupos: leves: índice I e 2 (menos que 3 vezes ao dia); intensas: 3 (de 3 a 10 vezes ao dia) e 4.

As variáveis independentes consideradas foram: idade, cor, escolaridade, estado marital, emprego, estrato social, região geográfica, idade à menarca, paridade, número de abortos, uso de método anticoncepcional (MAC) hormonal, MAC não hormonal, laqueadura tubária, IMC, uso de terapia hormonal $(\mathrm{TH})$, estado menopausal, tempo de menopausa, histerectomia, ooforectomia bilateral, hábito de fumar, número de cigarros e tempo de tabagismo.

O estado menopausal foi definido segundo Jaszmann?: pré-menopausa: ciclos menstruais regulares ou com padrão menstrual similar ao que elas tiveram durante a vida reprodutiva; perimenopausa: ciclos menstruais nos últimos 12 meses, mas com mudança do padrão menstrual quando comparado aos padrões anteriores; pós-menopausa: último período menstrual ocorreu havia pelo menos 12 meses.

$O$ estrato social foi definido utilizando-se os critérios da Associação Brasileira dos Anunciantes (ABA) e da Associação Brasileira dos Institutos de Pesquisa de Mercado (ABIPEME) e classificado em classe A (89 pontos ou mais); classe B (59-88 pontos); classe C (35-58 pontos); classe D (20-34 pontos); e classe $\mathrm{E}$ (0- 19 pontos).

Para análise dos dados utilizou-se o programa SAS, versão 8.2. Inicialmente as variáveis foram estudadas de maneira descritiva por meio do cálculo de freqüências absoluta e relativa e, no caso das variáveis contínuas, pelo cálculo da média, desvio padrão e mediana. Para estudar a associação da variável dependente com as variáveis independentes, utilizou-se a razão de prevalência (RP). Estimou-se a RP da intensidade das ondas de calor para cada variável independente. Estas razões foram comparadas entre as respectivas categorias de cada variável independente com intervalos de confiança de 95\% (IC 95\%). Em uma segunda etapa, foi realizada a análise de regressão múltipla, a fim de identificar quais variáveis estariam, conjuntamente, associadas à intensidade das ondas de calor.

O protocolo deste estudo foi aprovado pela Comissão de Pesquisa do Departamento de Tocoginecologia e Comitê de Ética em Pesquisa da Faculdade de Ciências Médicas da Universidade Estadual de Campinas.

\section{Resultados}

As participantes deste estudo apresentaram mediana de idade de 5 I anos. A idade, cor/etnia, escolaridade, estado marital, emprego, classe social e região geográfica não se associaram à intensidade das ondas de calor (Tabela I). 
Tabela I - Características sociodemográficas, segundo a intensidade das ondas de calor $(n=334)$

\begin{tabular}{|c|c|c|c|c|c|c|}
\hline \multirow{3}{*}{$\begin{array}{c}\text { Características } \\
\text { Idade (anos) }\end{array}$} & \multicolumn{4}{|c|}{ Ondas de calor } & & \\
\hline & \multicolumn{2}{|c|}{ Intensas $(n=|3|)$} & \multicolumn{2}{|c|}{ Leves $(n=203)$} & \multicolumn{2}{|c|}{ Análise bivariada } \\
\hline & $\mathbf{N}$ & $\%$ & $\mathbf{N}$ & $\%$ & RP* & IC 95\% \\
\hline$\leq 51$ & 65 & 49,6 & 92 & 45,3 & 1,00 & - \\
\hline$>51$ & 66 & 50,4 & 111 & 54,7 & 0,90 & $0,64-|, 4|$ \\
\hline \multicolumn{7}{|l|}{ Cor/etnia } \\
\hline Branca & 71 & 54,2 & 110 & 54,2 & 1,00 & - \\
\hline Não branca & 60 & 45,8 & 93 & 45,8 & 1,00 & $0,7|-|, 4 \mid$ \\
\hline \multicolumn{7}{|l|}{ Escolaridade } \\
\hline Até primário & 121 & 92,4 & 173 & 85,6 & 1,00 & - \\
\hline Secundário ou universitário & 10 & 7,6 & 29 & 14,4 & 0,62 & $0,33-1,19$ \\
\hline Sem resposta & 0 & & । & & & \\
\hline \multicolumn{7}{|l|}{ Estado marital } \\
\hline Casada/amasiada & 92 & 70,2 & 142 & 70,0 & 1,00 & - \\
\hline Solteira & 39 & 29,8 & 61 & 30,0 & 0,99 & $0,68-1,44$ \\
\hline \multicolumn{7}{|l|}{ Emprego } \\
\hline Não & 76 & 58,0 & 136 & 67,0 & 1,00 & - \\
\hline $\operatorname{Sim}$ & 55 & 42,0 & 67 & 33,0 & 1,26 & $0,89-1,78$ \\
\hline \multicolumn{7}{|l|}{ Classe Social } \\
\hline Classe Ae B & 18 & 13,8 & 40 & 19,7 & 1,00 & - \\
\hline Classe C & 56 & 42,7 & 82 & 40,4 & $|, 3|$ & $0,77-2,22$ \\
\hline Classe De E & 57 & 43,5 & 81 & 39,9 & 1,33 & $0,78-2,26$ \\
\hline \multicolumn{7}{|l|}{ Região } \\
\hline Sul e Sudeste & $|2|$ & 92,4 & 183 & 90,1 & 1,00 & - \\
\hline Norte e Nordeste & 8 & 6,1 & 16 & 7,9 & 0,84 & $0,4|-|, 7 \mid$ \\
\hline Centro-Oeste & 2 & 1,5 & 4 & 2.0 & 0.84 & $0,21-3,39$ \\
\hline
\end{tabular}

*RP: razão de prevalência; *IC95\%: intervalo de confiança de 95\%

A mediana de idade à menarca foi de 13 anos. Em relação às características reprodutivas, paridade, métodos contraceptivos hormonais e não hormonais não se associaram à intensidade das ondas de calor. A história de tabagismo não se associou à intensidade das ondas de calor.

Aproximadamente $59 \%$ das mulheres com ondas de calor intensas não haviam utilizado terapia hormonal e 48\% estavam na pós-menopausa. $\mathrm{Na}$ análise bivariada, o tempo de menopausa superior a 61 meses associou-se significativamente à ocorrência de ondas de calor de intensidade leve (RP: 0,49; IC 95\%: 0,28-0,85) (Tabela 2).

A análise conjunta das variáveis mostrou que mulheres com tempo de menopausa superior a 6 I meses (RP: 0,59; IC 95\%: 0,39-0,88) apresentaram chance significativamente menor de apresentar ondas de calor intensas, enquanto o antecedente de ooforectomia bilateral (RP: I,95; IC 95\%: I,08-3,50) associou-se significativamente à intensidade das ondas de calor (Tabela 3).

\section{Discussão}

O objetivo desse estudo foi identificar os fatores associados à intensidade das ondas de calor em mulheres climatéricas. Sabemos que os sintomas menopausais estão inter-relacionados, podendo ser classificados em componentes vasomotores e psicológicos. O componente vasomotor é composto por ondas de calor e sudorese e o psicológico por depressão, nervosismo e irritabilidade ${ }^{3}$ Entretanto, a literatura mostra que as ondas de calor, sintoma mais comum em mulheres ocidentais, têm a capacidade de gerar o efeito dominó, ou seja, um sintoma principal desencadeando outros componentes da síndrome climatérica.

Observou-se que o tempo de pós-menopausa inferior a 61 meses associou-se às ondas de calor de maior intensidade. Quando se realizou análise de regressão múltipla, ou seja, consideraram-se todas as variáveis em conjunto, além do tempo de menopausa, o antecedente de ooforectomia bilateral associou-se às ondas de calor mais intensas. Esses resultados estão de acordo com os relatados em mulheres australianas, nas quais a intensidade dos sintomas vasomotores aumentou na pós-menopausa ${ }^{6}$.

O início súbito das ondas de calor após a ooforectomia bilateral está relacionado à diminuição dos esteróides sexuais. Outros autores também relataram uma associação entre ooforectomia e ondas de calor de maior intensidade 4 . Quando a menopausa ocorre naturalmente, as alterações nos níveis circulantes de esteróides sexuais e do hormônio folículo estimulante são graduais, iniciando aproximadamente dois anos antes da última menstruaçãa ${ }^{10}$. Esta diferença no declínio dos níveis de esteróides sexuais entre a menopausa cirúrgica e a natural pode explicar a intensidade das ondas de calor determinada pela menopausa cirúrgica, como demonstrado no presente estudo. 
Tabela 2 - Características referentes à menopausa, segundo a intensidade das ondas de calor $(\mathrm{n}=334)$

\begin{tabular}{|c|c|c|c|c|c|c|}
\hline \multirow{3}{*}{$\begin{array}{l}\text { Características } \\
\text { Uso de terapia hormonal }\end{array}$} & \multicolumn{4}{|c|}{ Ondas de calor } & & \\
\hline & \multicolumn{2}{|c|}{ Intensas $(n=|3|)$} & \multicolumn{2}{|c|}{ Leves $(n=203)$} & \multicolumn{2}{|c|}{ Análise bivariada } \\
\hline & $\mathbf{N}$ & $\%$ & $\mathbf{N}$ & $\%$ & RP* & IC $95 \% \#$ \\
\hline Nuncausou & 77 & 58,8 & 115 & 56,6 & 1,00 & \\
\hline Usa & 30 & 22,9 & 45 & 22,2 & 1,00 & $0,65-1,52$ \\
\hline Usou & 24 & 18,3 & 43 & 21,2 & 0,89 & $0,57-|, 4|$ \\
\hline \multicolumn{7}{|l|}{ Estado menopausal } \\
\hline Pré-menopausa & 26 & 19,8 & 37 & 18,2 & 1,00 & - \\
\hline Perimenopausa & 19 & 14,5 & 26 & 12,8 & 1,02 & $0,57-1,85$ \\
\hline Pós-menopausa & 63 & 48,1 & 104 & 51,3 & 0,91 & $0,58-1,44$ \\
\hline Histerectomizada & 23 & 17,6 & 36 & 17,7 & 0,95 & $0,54-1,66$ \\
\hline \multicolumn{7}{|l|}{ Ooforectomia bilateral } \\
\hline Não & 116 & 89,9 & $|9|$ & 96,0 & 1,00 & \\
\hline $\operatorname{Sim}$ & 13 & $|0|$, & 8 & 4,0 & 1,64 & $0,93-2,91$ \\
\hline Nãosabe/nãolembra & 2 & - & 4 & - & & \\
\hline \multicolumn{7}{|c|}{ Tempo de menopausa (meses)*** } \\
\hline$\leq 6 i$ & 45 & 71,4 & 47 & 45,2 & 1,00 & - \\
\hline$>61$ & 18 & 28,6 & 57 & 54,8 & 0,49 & $0,28-0,85$ \\
\hline
\end{tabular}

*RP: razão de prevalência; ${ }^{*}$ IC95\%: intervalo de conflança de 95\%; **Somente mulheres na pós-menopausa

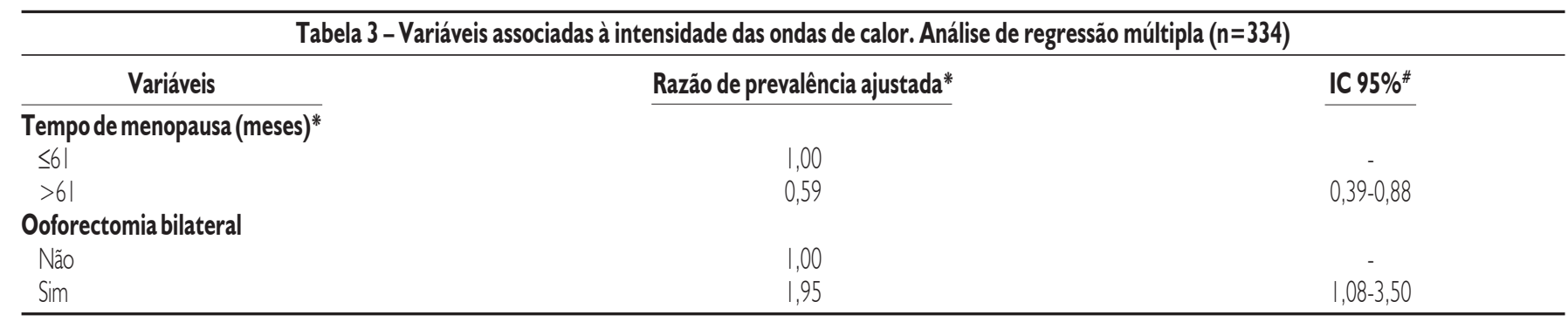

*Aiustada por idade, cor, escolaridade, estado marital, emprego, estrato social, região geográfica, idade à menarca, paridade, número de abortos, uso de método anticoncepcional hormonal e não hormonal, laqueadura tubária, índice de massa corpórea, uso de terapia hormonal, estado menopausal, tempo de menopausa, histerectomia, ooforectomia bilateral, hábito de fumar, número de cigarros e tempo de tabagismo. \#IC 95\%: intervalo de confiança de $95 \%$

Quanto à cor/etnia não se observou associação com a intensidade das ondas de calor, provavelmente pela dificuldade de avaliação dessa variável em nosso meio. Cabe ressaltar que, no presente estudo, essa variável foi classificada segundo autodeclaração da participante. Importante também destacar que o papel da cor/etnia nos níveis hormonais durante a transição climatérica é pouco conhecido. O estudo Study of Women's Health Across the Nation demonstrou que mulheres americanas afrodescendentes foram as que apresentaram os menores níveis de testosterona e de sulfato de deidroepiandrosterona quando comparadas às caucasianas, hispânicas e orientais. Possivelmente por este motivo, as afro-americanas foram as que reportaram maior prevalência de ondas de calor ${ }^{5}$.

Na presente casuística, não se observou relação entre intensidade das ondas de calor e histerectomia. Os dados da literatura são conflitantes quanto a essa associação. Oldenhave et al. ${ }^{2}$ mostraram que mulheres com antecedente de histerectomia referiram sintomas climatéricos mais intensos que aquelas não histerectomizadas. É importante salientar que nem todos os estudos observaram relação entre histerectomia e ocorrência e intensidade das ondas de calor ${ }^{\prime \prime, 4}$.
Outro fator cuja associação permanece controversa é o IMC. Li et al..$^{4}$ descreveram uma associação entre alto IMC e maior prevalência e intensidade das ondas de calor, fato observado por outros autores ${ }^{5}$. Porém, alguns autores relataram que mulheres com menor IMC apresentam maior prevalência de ondas de calor ${ }^{12,13}$. Schwingl et al. ${ }^{14}$ observaram que os efeitos de IMC baixo sobre o risco de apresentar ondas de calor estavam relacionados com o tabagismo, ou seja, o IMC baixo só associou-se às ondas de calor em mulheres tabagistas. Por outro lado, alguns estudos relataram aumento do risco de apresentar ondas de calor em mulheres com maior peso ${ }^{15}$. Outros pesquisadores não observaram associação entre IMC e ondas de calor ${ }^{16}$, em concordância com os nossos dados.

Nesse estudo, o tabagismo não se associou à intensidade das ondas de calor. O tabagismo pode reduzir os níveis circulantes de estrogênio por meio da interação com o sistema enzimático do citocromo P450. Esse sistema é responsável pelo metabolismo dos componentes químicos do fumo, bem como do estrogênio ${ }^{17}$. Cassidenti et al. ${ }^{18}$ mostraram que, em usuárias de estrogênio 
oral, o metabolismo hepático do estrogênio estava aumentado quando essas usuárias eram fumantes. Os componentes químicos do cigarro podem reduzir a conversão de androgênios para estrogênios devido à inibição da aromatase ${ }^{19}$, induzindo mutações ou destruindo os folículos ovarianos, ou ainda alterando indiretamente o metabolismo do estrogênio pela redução do peso corpóreo ${ }^{20}$.

O uso da terapia hormonal não influenciou a intensidade das ondas de calor. E provável que mulheres com ondas de calor intensas estivessem em uso da terapia hormonal no momento da entrevista. Verificou-se que usuárias e ex-usuárias apresentaram porcentagens semelhantes de ondas de calor intensas. Cabe destacar o grande porcentual $(58,8 \%)$ de mulheres que nunca utilizaram terapia hormonal e que relataram ondas de calor intensas, talvez demonstrando um equívoco na atenção à síndrome do climatério em nossa região. Um outro ponto que merece citação é que, nesta mesma casuística, o uso de terapia hormonal associou-se à maior escolaridade e melhor estrato social ${ }^{21}$, sendo uma possível justificativa para a não associação dessas variáveis com a intensidade das ondas de calor.

Finalmente, acreditamos que este estudo poderá contribuir para a melhor assistência às mulheres durante o climatério, uma vez que foram identificados fatores que se associaram independentemente à intensidade das ondas de calor. Mulheres com tempo de menopausa de até cinco anos ou com o antecedente de ooforectomia bilateral deverão ser alvo de intervenções que minimizem as repercussões destes fatores sobre as ondas de calor. Essas intervenções poderão incluir medidas farmacológicas e programas educacionais que esclareçam o conceito de menopausa e a melhor forma de vivenciar seus sintomas. Outro aspecto relacionase à mudança do estilo de vida, estimulando-se hábitos mais saudáveis como o abandono do tabagismo e do consumo de álcool e a prática de exercícios físicos. Certamente essas medidas podem minimizar a ocorrência e a intensidade das ondas de calor, contribuindo para uma melhor qualidade de vida.

Estudos prospectivos que se iniciem na pré-menopausa deverão avaliar a prevalência e a intensidade dos sintomas vasomotores, em especial das ondas de calor, e sua relação com a qualidade de vida. Deverão incluir também dosagens hormonais, ressaltando-se as concentrações séricas de estrogênios e androgênios e suas correlações com os sintomas psicológicos e vasomotores. A identificação dos fatores de risco para ondas de calor mais intensas contribuirá para a prevenção destes sintomas.

\section{Conclusão}

O tempo de menopausa inferior a cinco anos e o antecedente de ooforectomia bilateral associaram-se às ondas de calor intensas, sugerindo o hipoestrogenismo como causa comum para as ondas de calor de maior intensidade. Mulheres com esses fatores devem receber atenção especializada, procurando minimizar as repercussões negativas desse sintoma.

\section{Conflito de interesse: não há.}

\section{SUMMARY}

FACTORS ASSOCIATED WITH THE INTENSITY OF HOT FLASHES IN CLIMACTERIC WOMEN

OBIECTIVE. To identify factors associated with the intensity of hotflashes in 334 climacteric women living in Campinas, São Paulo, Brasil.

METHODS. Secondary analysis of a data bank of a descriptive crosssectional population-based study. Selection of 334 women reporting hot flashes aged 45-60 years was carried out through cluster sampling. Data were collected by home interviews using a structured, pre-tested questionnaire provided by the International Health Foundation/ International Menopause Society and by the North American Menopause Society and adapted by the authors. Intensity of hot flashes was measured using the circulatory index. The variables analyzed were age, use of contraceptive methods and hormonal therapy, tubal ligation, body mass index, menopausal status, time since menopause, hysterectomy, bilateral oophorectomyand smoking. Statistical analysis was performed by using the median, absolute and relative frequencies according to the type of variable. The prevalence ratio (PR) was used to measure association. Bivariate analysis and multiple logistic regression with a $95 \%$ confidence interval $(95 \% \mathrm{Cl})$ were used to identify the factors associated with the intensity of hot flashes.

RESULTS. Women with time since menopause of over 61 months (PR: 0.59; IC 95\%: 0.39-0.88) had a significantly lower chance of presenting intense hot flashes while the antecedent of bilateral oophorectomy (PR: 1.95; IC95\%: 1.08-3.50) was significantly associated with the intensity of hot flashes.

CONCLUSION. Both factors, time since menopause and bilateral oophorectomy, suggest hypoestrogenism as a common cause of more intense hot flashes. Women with these factors should receive specialized care to minimize the negative effects of hot flashes. [Rev Assoc Med Bras 2006; 52(6): 413-8]

KEY WORDS: Menopause. Cross-sectional studies. Hot flashes

\section{REFERÊNCIAS}

I. Kronenberg F, Downey JA. Thermoregulatory physiology of menopausal hot flashes: a review. Can J Physiol Pharmacol. 1987;65: I 312-24.

2. Oldenhave A, Jaszmann LJ, Haspels AA, Everaerd WT. Impact of climacteric on well being. Am J Obstet Gynecol. 1993; 168:772-80.

3. Pedro AO, Pinto-Neto AM, Costa-Paiva LHS, Osis MJD, Hardy EE. Síndrome do climatério: inquérito populacional domiciliar em Campinas, SP. Rev Saúde Publica. 2003;37:735-42.

4. Li C, Samsioe G, Borgfeldt C, Lidfeldt J, Agardh CD, Nerbrand C. Menopause-related symptoms: what are the background factors? A prospective population-based cohort study of Swedish women (The Women's Health in Lund Area study). Am J Obstet Gynecol. 2003; 1 89: 1 646-53.

5. Sherman S. Natural history of menopause studies and related efforts at the National Institute on Aging, NIH. In: Schneider HPG, Naftolin F, editors. Climacteric medicine - where do we go? London: Taylor \& Francis; 2005. p. 16-26.

6. Dennerstein L, Guthrie JR, Taffe JR, Lehert P, Burger HG. In: Schneider HPG, Naftolin F, editors. Climacteric medicine: where do we go? London: Taylor \& Francis; 2005. p.27-35.

7. Santos-Sá D, Pinto-Neto AM, Conde DM, Pedro AO, Oliveira SCM, Costa-Paiva LHS. Fatores associados às ondas de calor em mulheres climatéricas: inquérito populacional domiciliar. Rev Bras Ginecol Obstet. 2004;26:765-71. 
8. Boulet MJ, Oddens BJ, Lehert P, Vemer HM, Visser A. Climacteric and menopause in seven South-east Asian countries. Maturitas. 1994; 19:157-76.

9. Jaszmann L. Epidemiology of climacteric and post-climacteric complaints. In: Van Keep PA, Lauritzen C, editors. Ageing and estrogens 3rd ed. Front Hormone Res.. 1973;2:22-34.

10. Burger HG, Dudley EC, Hopper JL, Groome N, Guthrie JR, Green A, et al. Prospectively measured levels of serum follicle-stimulating hormone, estradiol, and the dimeric inhibins during the menopausal transition in a population-based cohort of women. J Clin Endocrinol Metab. 1999;84:4025-30

I I. Kritz-Silverstein D, Goldani Von Muhlen, D, Barret-Connor E. Prevalence and clustering of menopausal symptoms in older women by hysterectomy and oophorectomy status. J Womens Health Gender-Based Med. 2000;9:747-55.

12. Campagnoli C, Morra G, Belforte P, Belforte L, Prelato Tousijn L. Climacteric symptoms according to body weight in women of different socio-economic groups. Maturitas. 1981;3:279-87.

13. Erlik Y, Meldrum DR, Judd HL. Estrogen levels in postmenopausal women with hot flashes. Obstet Gynecol. 1982;59:403-7

I4. Schwingl PJ, Hulka BS, Harlow SD. Risk factors for menopausal hot flashes. Obstet Gynecol. 1994;84:29-34.

15. Whiteman MK, Staropoli C, Langenberg P, Mccarter R, Kjerulff K, Flaws JA. Smoking, body mass, and hot flashes in mid-life women. Obstet Gynecol. 2003; 101 : 264-72.
16. Staropoli CA, Flaws JA, Bush TL, Moulton AW. Predictors of menopausal hot flashes. J Womens Health. 1998; 7: I|49-55.

17. Avis NE, Crawford S, Stellato R, Longcope C. Longitudinal study of hormone levels and depression among women transitioning trough menopause. Climacteric. 2001;4:243-9.

18. Cassidenti DL, Vijod AG, Vijod MA, Stanczyk FZ, Lobo RA. Short-term effects of smoking on the pharmacokinetic profiles of micronized estradiol in postmenopausal women. Am J Obstet Gynecol. 1990; | 63: 1953-60.

19. Barbieri RL, Mcshane PM, Ryan KJ. Constituents of cigarette smoke inhibit human granulose cell aromatase. Fertil Steril. 1986;46:232-6.

20. Blumberg G, Kaplan B, Rabinerson D, Goldman GA, Kitai E, Neri A Women's attitudes towards menopause and hormone replacement therapy. Int J Gynaecol Obstet. 1996;54:27। -7.

21. Pinto-Neto AM, Pedro AO, Hardy E, Osis MJ, Costa-Paiva LH, Martinez EZ. Caracterização das usuárias de terapia de reposição hormonal do Município de Campinas, São Paulo. Cad Saude Publica. 2002; I 8: I 2 I-7.

Artigo recebido: 21/1 I/2005

Aceito para publicação: 27/03/2006 